\title{
Recent progress in the field of cIMP research
}

\author{
Roland Seifert $^{1}$
}

Received: 8 August 2016 / Accepted: 9 August 2016/Published online: 18 August 2016

(C) Springer-Verlag Berlin Heidelberg 2016

\section{Abbreviations \\ cIMP Inosine 3',5'cyclic monophosphate \\ 3',5'-cyclic cNMP Nucleoside 3',5'-cyclic monophosphate}

cGMP is an established second messenger generated by soluble guanylyl cyclase (sGC) and particulate guanylyl cyclases (pGCs) (Friebe et al., 2015). cGMP mediates its biological effects via protein kinase $\mathrm{G}$, ion channels and inhibition or stimulation of nucleoside 3',5'-cyclic monophosphate (cNMP)-degrading phosphodiesterases (Friebe et al., 2015). The GC-cGMP system is of substantial pharmacological interest, specifically for the treatment of cardiovascular diseases (Friebe et al., 2015). Probably the most important pharmacological effect of cGMP is to induce vasodilation (Friebe et al., 2015).

In vitro studies with highly purified sGC and sensitive and specific mass spectrometry methods have unequivocally shown that in addition to cGMP, sGC is also capable of effectively producing inosine $3^{\prime}, 5^{\prime}$ cyclic monophosphate (cIMP) (Beste et al., 2012). pGCs generate cIMP as well (Beste et al., 2013). Moreover, the "adenylyl cyclase" toxins CyaA from Bordetella pertussis and edema factor from Bacillus anthracis can generate cIMP (Beste et al., 2013). There is only a small chemical difference between cGMP and cIMP, i.e., cIMP misses an amino group at the purine ring (Fig. 1). As a consequence, cIMP is considerably less potent and effective than cGMP at activating protein kinase G (Wolter et al., 2011).

Roland Seifert

seifert.roland@mh-hannover.de

1 Institute of Pharmacology, Hannover Medical School, Carl-Neuberg-Str. 1, 30625 Hannover, Germany
cIMP belongs to the group of non-canonical cNMPs that also comprises cCMP and cUMP (Seifert et al., 2015).

Studies on various mammalian cell lines and mouse organs failed to detect cIMP (Beste et al., 2013; Hartwig et al., 2014; Bähre et al., 2015), raising doubts about a (patho)physiological role of cIMP (Seifert, 2014). However, the group of Vanhoutte showed that under conditions of hypoxia, sGC switches substrate specificity from GTP to ITP and that the generated cIMP exerts opposite (constrictory) effects on blood vessels to those of cGMP (dilatatory) (Chen et al., 2014; Gao and Vanhoutte, 2014). How such a small chemical difference between two cNMPs can result in opposite biological effects has been discussed controversially (Seifert, 2014; Gao and Vanhoutte, 2014).

On this background, some recent studies place the cIMP field into a new perspective. Specifically in rat kidney and rat liver, low levels of cIMP were detected using sensitive and specific mass spectrometry methods (Jia et al., 2014). In addition, several organs from zebrafish, i.e., eyes, heart and testes, contain cIMP (Dittmar et al., 2015). As a cautionary note, in these two studies, the identity of cIMP was not unequivocally confirmed by HPLC-time-of-flight mass spectrometry, the low abundance of cIMP and the relatively low sensitivity of timeof-flight mass spectrometry being limiting factors (Bähre et al., 2015; Dittmar et al., 2015). Clearly, these lacking data on cIMP must be provided. However, in aggregate, the available data suggest that cIMP occurs only under certain pathophysiological conditions and in certain species. Accordingly, a comprehensive survey of cIMP concentrations in multiple species and organs under various physiological and disease conditions is required. The zebrafish model is particularly well suited for studies on a potential role of cIMP in organ development.

In a recent study, $\mathrm{Ng}$ et al. (2016) systematically analyzed the interactions of canonical and non-canonical cNMPs with 


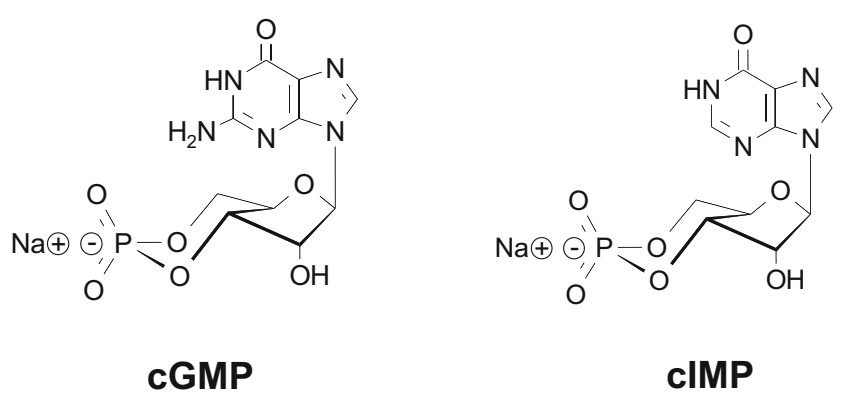

Fig. 1 Structures of cGMP and cIMP

the hyperpolarization-activated cNMP-gated channel 2 (HCN2). In contrast to the prototypical HCN2 channel activator cAMP, cIMP failed to induce oligomerization of the cNMP-binding domains of the channel and opened ion conductance only ineffectively. Crystallographic studies revealed that cIMP and cAMP stabilize distinct HCN2 channel conformations. The study of $\mathrm{Ng}$ et al. (2016) suggests that various cNMPs interact differentially with HCN channels, opening the door for specific pharmacological modulation of $\mathrm{HCN}$ channel subtypes in terms of full and partial activators and inhibitors.

In an unrelated recent study, the group of Vanhoutte followed up their previous study on cIMP-induced vasoconstriction (Chen et al., 2014) and examined the effects of thymoquinone on blood vessels (Detremmerie et al., 2016). Thymoquinone is the pharmacologically active constituent of Nigella sativa and exhibits pleiotropic pharmacological effects including antioxidant properties (Amin and Hosseinzadeh, 2016). These properties have been proposed to be of potential value for the treatment of a vast number of diseases including neurodegenerative disease, cancer, pain, diabetes and asthma (Amin and Hosseinzadeh, 2016; Elmaci and Altinoz, 2016; Gholamnezhad et al., 2016). However, convincing and wellcontrolled clinical studies are still missing. In their study, Detremmerie et al. (2016) show that thymoquinone at moderately high concentrations $(10-100 \mu \mathrm{M})$ can exhibit paradoxical vasoconstrictory effects via $\mathrm{Ca}^{2+}$ influx by T-type- or L-type $\mathrm{Ca}^{2+}$ channels. cIMP-induced vasoconstriction may play a role in coronary ischemia/reperfusion injury and/or cardiovascular complications in sleep apnea (Detremmerie et al., 2016). The occurrence of cIMP in only certain pathophysiological settings could be an explanation for the failure to detect cIMP in several biological systems under normal conditions, i.e., without hypoxia (Beste et al., 2013; Hartwig et al., 2014; Bähre et al., 2015).

Detremmerie et al. (2016) provide evidence in favor of the notion that the pharmacological actions of thymoquinone depend on sGC-catalyzed cIMP formation. Hence, thymoquinone constitutes a novel pharmacological tool that can be used to increase intracellular cIMP concentrations. The precise molecular mechanism of action of thymoquinone in the sGC-cGMP/cIMP system is not yet known, and it is unlikely that the pleiotropic pharmacological effects of the natural product (Amin and Hosseinzadeh, 2016) can all be attributed to a single molecular mechanism. To this end, it is clear that for the cIMP increase, the quinone moiety of thymoquinone is required (Detremmerie et al., 2016), a first step towards the elucidation of structure/activity relationships.

Notably, even extracellularly applied cIMP exhibits vasoconstrictory effects (Chen et al., 2014; Detremmerie et al., 2016). cIMP may either be taken up into cells via transporters and exert intracellular effects or may act extracellularly, e.g., by metabolism to inosine and subsequent activation of adenosine receptors (Chen et al., 2014; Seifert et al., 2015; Sassi et al., 2014; Detremmerie et al., 2016). Intracellular effects of cIMP are probably transient because several phosphodiesterases effectively hydrolyze cIMP (Monzel et al., 2014). It is also likely (but formally not yet proven) that cIMP is effectively transported via multidrug resistance proteins 4 and 5 (Laue et al., 2014). A regulatory role of cIMP on phosphodiesterase activity is possible as well (Monzel et al., 2014).

Thus, recent studies, despite initial skepticism (Seifert, 2014), support the notion that cIMP may exhibit a signaling role independently of that of cGMP. In order to further test this intriguing hypothesis, several lines of investigations must be pursued. Firstly, systematic pharmacological analyses of canonical and non-canonical cNMPs on every known purified cNMP effector protein are necessary to reveal full agonism, partial agonism, or antagonism of a given cNMP. Secondly, systematic crystallographic studies with cNMPs on known target proteins are required. Such studies provide the rational basis for the design of selective modulators of a given effector protein. Thirdly, studies with intact cells and organs need to be expanded. Particularly, a role of cIMP in brain diseases associated with hypoxia such as stroke, epilepsia, and brain trauma must be explored (Thompson and Ronaldson, 2014).

The available evidence suggests that the occurrence and the effects of cIMP may be species-specific (Jia et al., 2014; Dittmar et al., 2015; Detremmerie et al., 2016). Thus, numerous species beyond rat, mouse, rat, and human should be studied. Such studies should not only include membranepermeable cNMP-acetoxymethyl esters (Beckert et al., 2014) as pharmacological tools but also the native cNMPs that can exert biological effects themselves or via degradation products (Chen et al., 2014; Sassi et al., 2014; Seifert et al., 2015; Detremmerie et al., 2016). Furthermore, the precise molecular mechanism underlying the cIMP-increasing effects of the natural compound thymoquinone must be elucidated. Specifically, it must be assessed whether thymoquinone alters only the concentrations of cIMP or also the concentrations of other cNMPs. Additionally, the precise link between thymoquinone and sGC must be explored. Based on the present study of Detremmerie et al. (2016), adverse rather than beneficial effects of thymoquinone would be expected in intact organisms. Particularly, it needs to be examined whether 
thymoquinone deteriorates the outcome of experimental myocardial infarction. Conversely, compounds that prevent cIMP formation may be of therapeutic value in hypoxic conditions.

In conclusion, the pharmacological analysis of the sGCcGMP/cIMP-thymoquinome system offers several intriguing opportunities for a better understanding of hypoxia-associated diseases and the development of novel therapeutic strategies. Like many other natural compounds (Gach et al., 2015; Hassani et al., 2016) thymoquinone exerts pleiotropic effects and does probably not possess a single defined molecular mechanism of action (Amin and Hosseinzadeh, 2016). Thus, the search for potent and selective compounds that can increase cIMP concentrations must continue.

\section{References}

Amin B, Hosseinzadeh H (2016) Black cumin (Nigella sativa) and its active constituent, thymoquinone: an overview on the analgesic and anti-inflammatory effects. Planta Med 82:8-16

Bähre H, Hartwig C, Munder A, Wolter S, Stelzer T, Schirmer B, Beckert U, Frank DW, Tümmler B, Kaever V, Seifert R (2015) cCMP and cUMP occur in vivo. Biochem Biophys Res Commun 460:909-914

Beckert U, Grundmann M, Wolter S, Schwede F, Rehmann H, Kaever V, Kostenis E, Seifert R (2014) cNMP-AMs mimic and dissect bacterial nucleotidyl cyclase toxin effects. Biochem Biophys Res Commun 451:497-502

Beste KY, Burhenne H, Kaever V, Stasch JP, Seifert R (2012) Nucleotidyl cyclase activity of soluble guanylyl cyclase $\alpha_{1} \beta_{1}$. Biochemistry 51: 194-204

Beste KY, Spangler CM, Burhenne H, Koch H, Koch KW, Shen Y, Tang WJ, Kaever V, Seifert R (2013) Nucleotidyl cyclase activity of particulate guanylyl cyclase A: comparison with particulate guanylyl cyclases E and F, soluble guanylyl cyclase and bacterial adenylyl cyclases CyaA and edema factor. PLoS One 8:e70223

Chen Z, Zhang X, Ying L, Dou D, Li Y, Bai Y, Liu J, Liu L, Feng H, Yu X, Leung SWS, Vanhoutte PM, Gao Y (2014) cIMP synthesized by sGC as a mediator of hypoxic contraction of coronary arteries. Am J Physiol Heart Circ Physiol 307:H328-H336

Detremmerie CM, Chen Z, Li Z, Alkharfy KM, Leung SWS, Xu A, Gao Y and Vanhoutte PM (2016) Endothelium-dependent contractions of isolated arteries to thymoquinone require biased activity of sGC with subsequent cIMP production. J Pharmacol Exp Ther (in press)

Dittmar F, Abdelilah-Seyfried S, Tschirner SK, Kaever V (2015) Temporal and organ-specific detection of cNMPs including cUMP in the zebrafish. Biochem Biophys Res Commun 468:708-712

Elmaci I, Altinoz MA (2016) Thymoquinone: an edible redox-active quinone for the pharmacotherapy of neurodegenerative conditions and glial brain tumors. A short review. Biomed Pharmacother 83: $635-640$

Friebe A, Sandner P, Seifert R (2015) From bedside to bench-meeting report of the 7th international conference on cGMP "cGMP: generators, effectors and therapeutic implications" in Trier, Germany, from June 19th to 21st 2015. Naunyn Schmiedeberg's Arch Pharmacol 388:1237-1246

Gach K, Dlugosz A, Janecka A (2015) The role of oxidative stress in anticancer activity of sequiterpene lactones. Naunyn Schmiedeberg's Arch Pharmacol 388:477-486

Gao Y, Vanhoutte PM (2014) Tissues cIMPly do not lie. Naunyn Schmiedeberg's Arch Pharmacol 387:901-903

Gholamnezhad Z, Havakhah S and Boskabady MH (2016) Preclinical and clinical effects of Nigella sativa and its constituent, thymoquinone: a review. J Ethnopharmacol (in press)

Hartwig C, Bähre H, Wolter S, Beckert U, Kaever V, Seifert R (2014) cAMP, cGMP, cCMP and cUMP concentrations across the tree of life: high cCMP and cUMP levels in astrocytes. Neurosci Lett 579: 183-187

Hassani FV, Shirani K and Hosseinzadeh H (2016) Rosemary (Rosmarinus officinalis) as a potential therapeutic plant in metabolic syndrome. Naunyn-Schmiedbergs Arch Pharmacol (in press)

Jia X, Fontaine BM, Strobel F, Weinert EE (2014) A facile and sensitive method for quantification of cyclic nucleotide monophosphates in mammalian organs: basal levels of eight cNMPs and identification of 2',3'-cIMP. Biomolecules 4:1070-1092

Laue S, Winterhoff M, Kaever V, van den Heuvel JJ, Russel FG, Seifert R (2014) cCMP is a substrate for MRP5. Naunyn Schmiedeberg's Arch Pharmacol 387:893-895

Monzel M, Kuhn M, Bähre H, Seifert R, Schneider EH (2014) PDE7A1 hydrolyzes cCMP. FEBS Lett 588:3469-3474

Ng L, Putrenko I, Baronas V, Van Petegem F and Accili EA (2016) Cyclic purine and pyrimidine nucleotides bind to the HCN2 channel and variably promote $\mathrm{C}$-terminal domain interactions and opening. Structure (in press)

Sassi Y, Ahles A, Truong DJ, Baqi Y, Lee SY, Husse B, Hulot JS, Foinquinos A, Thum T, Müller CE, Dendorfer A, Laggerbauer B, Engelhardt S (2014) Cardiac myocyte-secreted cAMP exerts paracrine action via adenosine receptor activation. J Clin Invest 124: 5385-5397

Seifert R (2014) Is cIMP a second messenger with functions opposite to those of cGMP? Naunyn Schmiedeberg's Arch Pharmacol 387: 897-899

Seifert R, Schneider EH, Bähre H (2015) From canonical to noncanonical cyclic nucleotides as second messengers: pharmacological implications. Pharmacol Ther 148:154-184

Thompson BJ, Ronaldson PT (2014) Drug delivery to the ischemic brain. Adv Pharmacol 71:165-202

Wolter S, Golombek M, Seifert R (2011) Differential activation of cAMPand cGMP-dependent protein kinases by cyclic purine and pyrimidine nucleotides. Biochem Biophys Res Commun 415:563-566 